\title{
Prosthetic Considerations in Oral and Maxillofacial Trauma
}

\author{
Sudhir Bhandari
}

\begin{abstract}
Intraoral trauma may occur in isolation or in combination with injury of the other parts of the body. Once the life-threatening phase of trauma is over, the primary need for the patient is to restore the form and function of the tissues lost due to trauma. In the oral and maxillofacial region, esthetic consideration does sometimes supersede the need of function. This article briefly summarizes the scope of prosthodontics in patients with oral and maxillofacial trauma.
\end{abstract}

Keywords: Oral and maxillofacial, Prosthesis, Traum.

How to cite this article: Bhandari S. Prosthetic Considerations in Oral and Maxillofacial Trauma. J Postgrad Med Edu Res 2014; 48(2):87-90.

\section{Source of support: Nil}

Conflict of interest: None

\section{INTRODUCTION}

Intraoral trauma occurs in isolation or in combination with maxillofacial trauma and other serious body injuries. Along with the expense involved in rehabilitation; patients afflicted with trauma bear the burden of temporary or lifelong morbidity. ${ }^{1}$ When limited to the trauma of oral and maxillofacial region the morbidity may range from as simple as fracture of a part of tooth structure and the injury may have an impact lasting for life.

Glossary of prosthodontics terms ${ }^{2}$ defines trauma as an injury or wound, whether physical or psychic. Extensive trauma especially in the maxillofacial region involve both, however the latter component dominates once the physical aspect of the injury is taken care of.

Goal of a prosthodontist is the perpetual preservation of what is present, achieving the realistic treatment goals and maintaining the outcome which should be acceptable to the patient and family. While achieving and maintaining satisfactory treatment results for trauma in maxillofacial rehabilitation; esthetic and functional outcomes must be considered when determining the proper treatment method. ${ }^{3}$ Loss of hard and soft tissue both intraoral and extraoral in

\footnotetext{
Assistant Professor

Oral Health Sciences Centre, Postgraduate Institute of Medical Education and Research, Chandigarh, India

Corresponding Author: Sudhir Bhandari, Assistant Professor Oral Health Sciences Centre, Postgraduate Institute of Medical Education and Research, Chandigarh, India, Phone: 0172-2626159, e-mail: docsudhirbhandari@gmail.com
}

patients with extensive trauma lead to marked compromised intraoral function and loss of an important facial organ and subsequent facial disfigurement. Once the acute phase of trauma is over and remaining tissues have stabilized, the primary concern for the patient is restoring the lost function and esthetics by means of intraoral and/or extraoral prosthesis.

\section{Prosthetic Compensations}

Choice of the intraoral prosthesis depends upon the amount of lost hard and soft tissue and the quality and quantity of the remaining supporting tissues. The compensation for the lost tissues is feasible by using gingival colored ceramics and heat cure polymethylmethacrylates denture bases (acrylic resin) in respectively based prosthesis (Figs 1A and B). Both materials can further be stained to match with the patient's gingival and skin color and pigmentation.

Extraoral prosthesis may be fabricated with silicones alone or a combination of silicone and acrylic resin. Silicones can further be internally colored and/or externally stained to achieve normal skin tone and color for a pleasing facial appearance.

\section{Need for Prosthetic Compensation}

1. When the defect is large enough to correct it with patient's own tissues.

2. When surgical effort to correct the tissue deficiencies has failed.

3. Patient does not give consent for the corrective procedures.

\section{Limitations}

1. Oral hygiene procedures are difficult in fixed restoration for extensive defects.

2. Food accumulation in resin based prostheses.

3. Wear and color change of resin and silicone based prostheses.

\section{Treatment Considerations}

A flow chart depicting the scope of intraoral prosthetic treatment options (Flow Chart 1). 


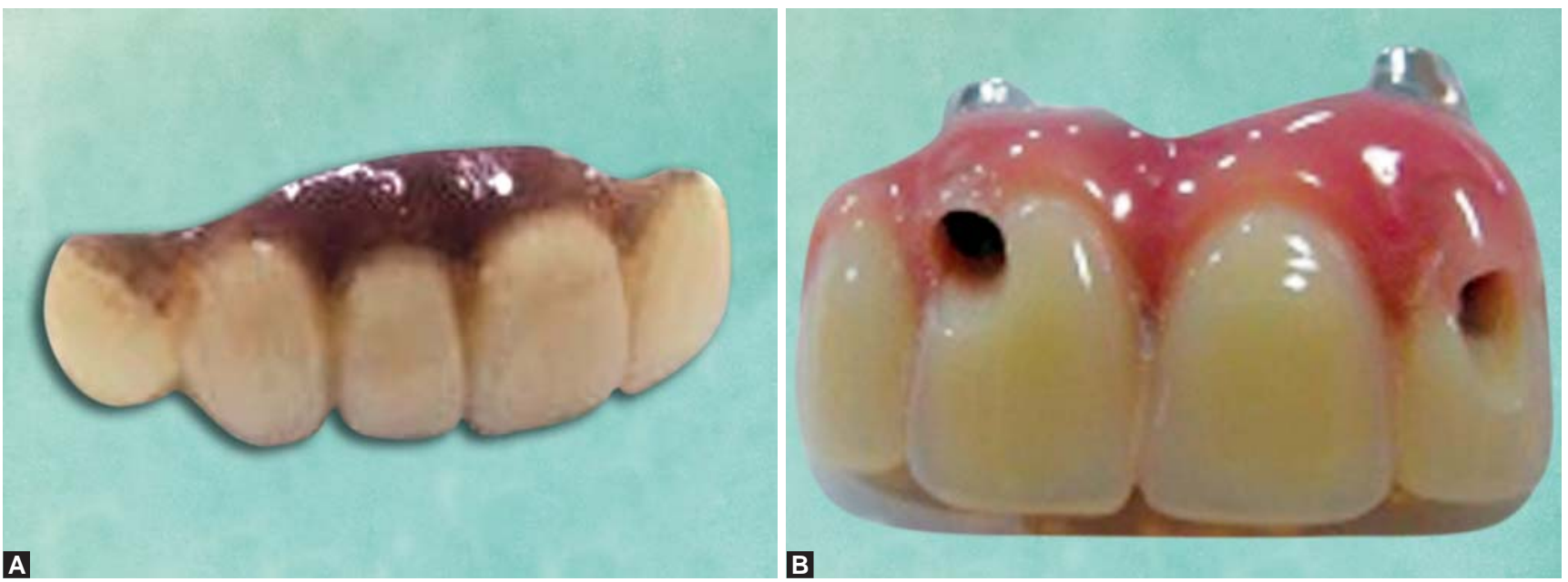

Figs $1 \mathrm{~A}$ and $\mathrm{B}$ : (A) Compensation of lost tissues using gingival ceramic which is further stained for color compatibility with adjacent gingiva, (B) compensation of lost tissue using heat cure acrylic resin in extensive tissue loss

\section{Intraoral Prosthetic Considerations}

Following prosthetic options are feasible depending on the clinical situation:

Treatment 1 (Tooth Concussion): Root canal treatment followed by full coverage restoration is the treatment of choice. Porcelain fused to metal crown or an all ceramic restoration may be the choice of material influenced by the region of the mouth involved, biomechanical requirement of the tooth, patient preferences and economics.

Treatment 2: Tooth loss without soft tissue deficiency.

Adjacent teeth (primary abutments) may require root canal treatment due to concussion or extensive material loss, then a tooth supported fixed partial denture may be the conventional treatment of choice. However, individual full coverage restoration for teeth and implant supported restoration for missing tooth/teeth is the more appropriate treatment option.

Flow Chart 1: Depicting the scope of intraoral prosthetic treatment options

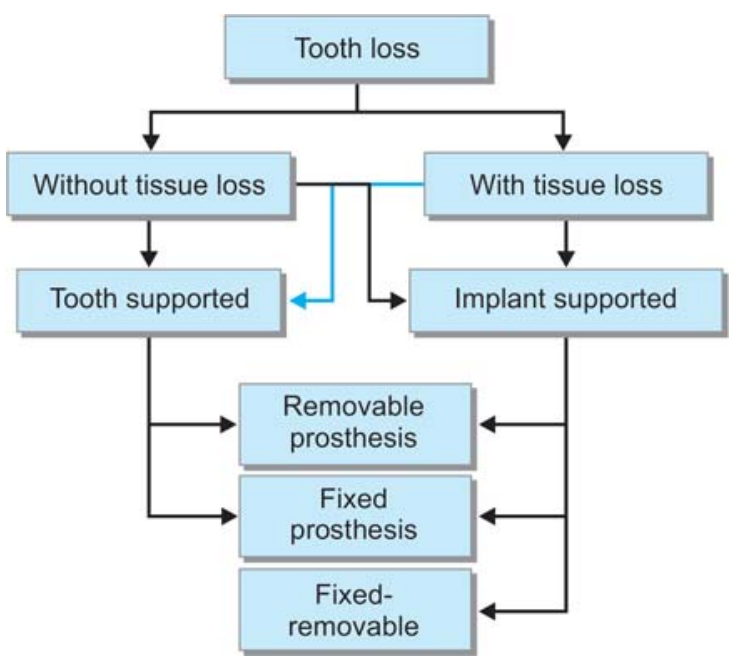

For tooth supported fixed dental prostheses in the esthetic zone, ovate pontic is the most desirable shape of the tooth to be replaced. It is a convex shaped pontic at its apex, extending 3 to $4 \mathrm{~mm}$ inside the free gingival margin, giving the natural emergence profile to the prosthetic replacement.

Immediate treatment options following traumatic loss of tooth: depending upon the clinical situation, a transient option of replacing a traumatic loss of tooth in the esthetic zone may be immediately replaced by patient's own tooth after modification or using an artificial acrylic resin tooth bonded to the adjacent teeth. ${ }^{4}$

A more permanent option would be to place immediate implant followed by a temporary prosthesis which may or may not be functionally loaded immediately to occlusal forces. Case selection and meticulous treatment planning is necessary to have a successful outcome in such a scenario.

Treatment 3: Tooth loss with soft and hard tissue deficiency.

Fixed dental prosthesis may involve use of gingival colored ceramic to compensate for the loss intraoral tissue. Custom staining is possible to have an acceptable final outcome (Figs 2A and B). However, ceramic being a brittle material has a limited utility in cases where extensive loss of intraoral tissue has occurred. To maintain the optimal facial contours, muscle support and tonicity; acrylic resin based prosthesis is a more prudent choice of material in patients presenting with extensive intraoral defects.

\section{Implant-supported Prosthesis}

With loss of tissues; if underlying quality and quantity of bone permits; an implant supported fixed acrylic resin based prosthesis (Fixed-removable) may be provided to the patient. It is important to have a thorough presurgical evaluation of the patient for soft and hard tissue grafting during and after 
implant surgery to achieve an acceptable and more importantly maintainable prosthetic outcome.

When implant supported removable prosthesis is the treatment of choice, it is important to choose from a variety of mechanical attachments for retaining the prosthesis. Choice of the attachment should be more objective rather than subjective and should depend upon amount of retention required, vertical dimension of the patient, interocclusal space available, number of implants, manual dexterity of the patient to maintain oral hygiene and longevity of the attachment components.

When rehabilitation with implant supported prosthesis is ruled out due lack of satisfactory intraoral foundation and biomechanical considerations; acrylic resin and/or cast partial denture based removable prosthesis should be given to the patient. Such prosthesis may be functionally deficient as compared to tooth or implant supported prosthesis but serves well to perform basic functions of masticatory apparatus and provide satisfactory esthetics.
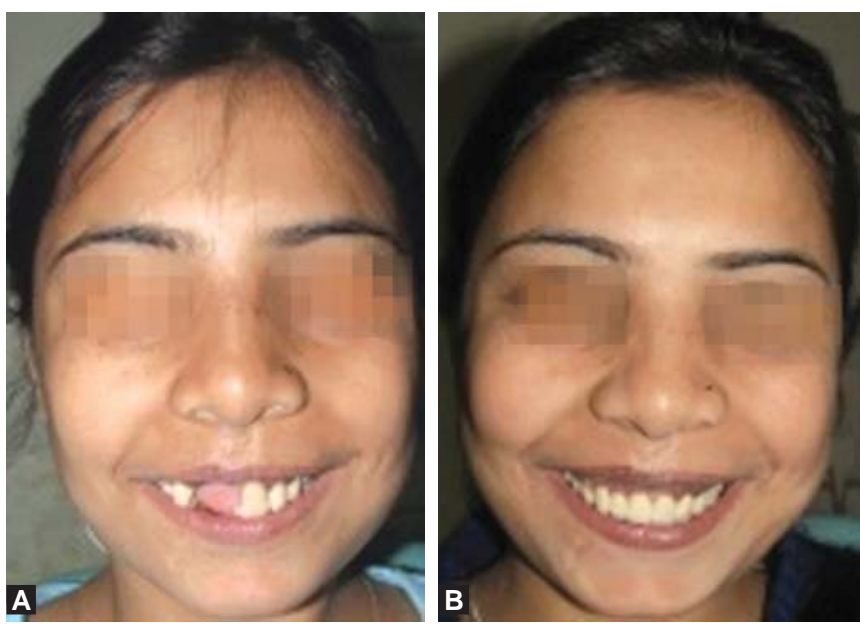

Figs 2A and B: (A) Preoperative view of lost anterior teeth with tissue deficiency and (B) postprosthetic cementation with gingival ceramic to compensate for the loss of tissues

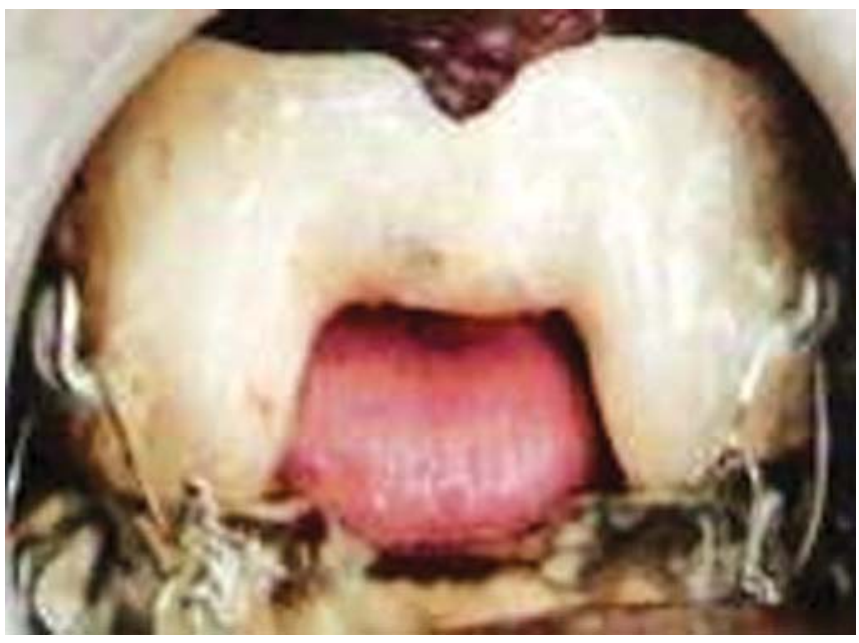

Fig. 3: Gunning splint with anterior opening for feeding and hooks for intermaxillary fixation

\section{Prosthetic Splints}

Treatment of fractured jaw(s) in completely edentulous patients who do not have a complete denture, may be done with the aid of Gunning splints. ${ }^{5}$ This appliance is fabricated on a cast which is sectioned and corrected at a determined vertical dimension specific to the patient. It is used to stabilize the jaws by aiding in intermaxillary fixation. Opening in the anterior region is given to aid in feeding and hooks are attached for the wires to be placed (Fig. 3). Overall oral hygiene maintenance and also around the hooks should be taught and reinforced at follow-up appointments.

\section{Extraoral Prosthetic Considerations}

Extensive defects in the region of the ear, nose or eye socket as a result of trauma require satisfactory cosmetic reconstruction to enable the patient to achieve full psychosocial and occupational rehabilitation and integration as early as possible. Spectacle, glue, bands and dental implant may be used for retaining such prosthesis. ${ }^{6}$

Prosthetic reconstruction has now become an established alternative to techniques using autogenous tissue. This can be a viable option, particularly for patients who are not willing to undergo major plastic surgery which sometimes requires several operations or in whom such procedures are contraindicated. $^{7,8}$

\section{DISCUSSION}

Scope of prosthesis in patients afflicted with trauma is immense. The necessity of cooperation of different medical specialists is inevitable before the patient reports for prosthetic rehabilitation. The goal of all involved in the rehabilitation of such patients is to improve their health related quality of life by facilitating the basic function of maxillofacial region like biting and swallowing, speech, esthetic rehabilitation. Re-establishing esthetics and function is the primary concern with underlying desire to feel being acceptable to self and society at large. However, patients expectations post trauma is usually guarded and with loss of hard and soft tissue in the oral and maxillofacial region the patients do realize that re-establishing the original form and function is not always possible. Prosthetic rehabilitation of such complex trauma, still remains a challenge as the emotional consequences of such trauma for patients with regard to possible long-term disfigurement needs to be addressed too. In such cases, an artificial replacement of an absent part of human body, i.e. prosthesis is deemed necessary. 
The ultimate aim, success does not necessarily require that the tooth is healthy and functioning for the entire life of the patient. Maintaining the tooth and surrounding bone for a few years can be considered a successful treatment in the growing patient.

Longevity of the treatment still remains an area of concern. Material failure and biologic failures may create a situation where the previous prosthesis is unsalvageable. In addition, failure to achieve an acceptable esthetics result may be a major cause of failure especially in the oral and maxillofacial area.

\section{CONCLUSION}

Achieving and maintaining an acceptable prosthetic replacement of lost tissues in oral and maxillofacial region is a rewarding experience. In addition to function and esthetics it bears a strong psychologic component and may have a major influence on the quality of life of the patient. However, the material characteristics and their inherent deficiencies affect the longevity of the treatment provided.

\section{REFERENCES}

1. Kraft A, et al. Craniomaxillofacial trauma synopsis of 14654 with 35,129 injuries in 15 years. Craniomaxillofac Trauma Reconstruction 2012;5:41-50.

2. Glossary of prosthodontic terms. J Prosthetic Dent 2005;94(1): 13-92.

3. Olsburgh S, Jacoby T, Krejci I. Crown fractures in the permanent dentition: pulpal and restorative considerations. Dent Traumatol 2002;18:103-115.

4. Bhandari S, Chaturvedi R. Natural tooth pontics: a viable yet temporary treatment option: a patient reported outcome. Indian J Dent Res 2012;23(2):59-63.

5. Brady FA, Tupac RG. Prosthodontic implications of oral and maxillofacial surgery. In: Maxillofacial rehabilitation: prosthodontics and surgical considerations. Beumer J, Curtis TA, Firtell DN, editors. CV Mosby Company. p 413-415.

6. Beumer J, Zlotolov I. Restoration of facial defects. Maxillofacial rehabilitation: prosthodontics and surgical considerations. In: Beumer J, Curtis TA, Firtell DN, editors. CV Mosby Company. p. 311-367.

7. Parel S, Tjellstrom A. The United States and Swedish experience with osseointegration and facial prostheses. Int J Oral Maxillofac Impl 1991;6:75-79.

8. Jacobsson M, Albrektsson T, Tjellstrom A. Tissue-integrated implants in children. Int J Ped Otorhinolaryngol 1992;24:235-243. 